\title{
The Construction and Practice of Internet Hospital in Hospital J
}

\author{
Feifei Guo, Fangzhou Liang* \\ The First Affiliated Hospital of Jinan University, Guangzhou, China \\ Email address: \\ guofeifeijnu@163.com (Feifei Guo), liangfangzhoujnu@163.com (Fangzhou Liang) \\ ${ }^{*}$ Corresponding author
}

\section{To cite this article:}

Feifei Guo, Fangzhou Liang. The Construction and Practice of Internet Hospital in Hospital J. World Journal of Public Health. Vol. 6, No. 2, 2021, pp. 40-44. doi: 10.11648/j.wjph.20210602.13

Received: April 27, 2021; Accepted: May 12, 2021; Published: May 21, 2021

\begin{abstract}
Background With the increasing demand for diversified health of the public and the increasingly mature technology of "Internet + medical treatment", the online business of hospitals continues to extend and expand. Under this background, Internet hospitals have witnessed rapid development in China in recent years. Objective In order to provide better patient services and improve the accessibility of quality medical services, Hospital J created Internet hospital to provide online diagnosis and treatment services. Methods Relying on Internet technology, Hospital $\mathrm{J}$ built hospital Internet online medical services, such as online registration, real-time consultation, message consultation, revisit prescription, drug distribution, pharmacist online, nursing consultation, appointment nurse home care and other services, supported by the offline hospital medical resources. Results Internet hospitals can help save the cost and time of patients' medical treatment, optimize the process of patients' medical treatment, avoid delay of illness and cross-infection, effectively alleviate the difficulty of patients' medical treatment, and improve the quality and level of hospital services. Conclusion In practice, Hospital J has explored a way to develop an Internet hospital in line with the characteristics of the hospital. Fundamentally, the development of Internet + medical treatment is fundamentally a change in the mode of doctor-patient service. With the advantages of convenience, efficiency and timeliness, it has become the trend of The Times. It is of positive practical significance to research on and solve the problems with the Internet hospital.
\end{abstract}

Keywords: Internet Hospital, Internet +, Online Diagnosis and Treatment, Medical Services

\section{Introduction}

With the continuous development of China's economy, the public's demand for medical services is growing day by day, so Internet + medical treatment emerges as The Times require and continues to grow in the process of exploration. The development of Internet hospital has experienced a process from nothing to existence, from scattered to standard, and from trial to standard [1]. Based on the continuous development of information technology, Internet telemedicine is becoming more and more mature. With the help of the Internet technology to promote health care reform, universal sharing has become a consensus of medical resources, all kinds of Internet + medical policies become introduced gradually in recent years. The construction of Internet + wisdom hospital is a response to the national health policy, improving residents medical treatment service, An effective means to realize "run once at most" medical service [2, 3]. In April 2018, the executive meeting of the State Council deliberated and adopted the Opinions on Promoting the Development of "Internet + Medical and Health" in principle. The Opinions pointed out that in terms of the development of "Internet + medical services", it is clearly stipulated that the development of Internet hospitals is allowed to rely on medical institutions, which can rely on hospitals and use Internet hospitals as the second name [4]. With the continuous development of hospitals, how to better serve patients and how to more conveniently facilitate residents to seek medical treatment has become a problem that must be solved at this stage [5]. Different from traditional hospitals, Internet hospitals can efficiently, quickly and safely provide the public with some diagnosis and treatment services independent of hospitals. The application of Internet hospitals is an important measure to improve medical services and build 
smart hospitals, as well as an important assessment index for the performance evaluation of third-level public hospitals [6].

At present, China's mainstream Internet hospital models can be divided into two types: the first is medical institutions as the main body; the second is based on Internet medical enterprises [7]. As one of the first batch of Internet hospitals in Guangdong Province, the Internet Hospital of Hospital $\mathrm{J}$ is belongs to the first model. It was officially launched and put into operation in June 2020, and incorporated the construction of Internet hospital into its overall development strategic plan. In order to provide better patient services, Hospital J plans to rely on Internet technology to create better online hospital diagnosis and treatment services, and fully tap the medical resources of the hospital to provide more residents with quality medical services [8]. Internet hospital services in general hospitals need the support of Internet hospital information platform. In order to realize the comprehensive coordination and homogenized medical quality management of online and offline medical services in the hospital, the hospital where the author works has built an online and offline integrated Internet hospital cloud platform. Since its operation, Hospital J has actively carried out the construction of Internet hospital, continuously improved the hospital's "Internet +" information construction level, improved the hospital's service quality and level, provided patients with a more comfortable medical experience, and explored a development road of Internet hospital in line with the hospital's characteristics in practice. This study proceeded from the background of the Internet hospital and the practical situation of Hospital J, expounds the application and construction of Internet hospitals in public general hospitals in the new era, and puts forward some thoughts and prospects for the development of Internet hospitals.

\section{The Construction of Internet Hospital in Hospital $\mathbf{J}$}

\subsection{Overall Situation}

Hospital $\mathbf{J}$ relying on Internet technology, built hospital Internet online medical services, supported by offline hospital medical treatment resources, The Internet hospital carries out "zero contact" in seeing a doctor and get medicine mode, implemented one-stop medical services services for patients, at the same time to effectively reducing hospital cross infection risk. In the hospital medical couplet body plays a demonstration role. The Internet Hospital of Hospital $\mathbf{J}$ has opened the following functions: online registration, real-time consultation, message consultation, revisit prescription, drug distribution, pharmacist online, nursing consultation, appointment nurse door-to-door care and other services. Some 130 doctors have been recruited online, covering 35 specialized departments, including 80 active doctors and 5 pharmacists, and 20 30 doctors/day have been received online. The Internet Hospital of Hospital $\mathrm{J}$ has been in trial operation since June 2020 and officially operated in October of the same year. As of March 2021, the cumulative number of consultations has reached 35,600 .

1) Patient side: Patients enter the official account or APP, register and log in, bind the hospital's medical card, and make a registration or appointment. When registering, patients need to fill in the relevant information of the patient, condition description, relevant pictures, report materials, etc. After the registration is successful and the registration fee is paid, parients wait for the doctor to initiate the video consultation. After the video communication, patients can check the consultation or diagnosis suggestions (including diagnosis, diagnosis opinions, outpatient medical records, prescriptions, etc.) given by doctors, and give satisfaction evaluation on the doctors and hospital services.

2) Doctor side: Doctors can log on to the medical page within the prescribed work schedule of the hospital or personal work schedule, and they can see the list of video consultation patients in the department and the list of video consultation patients to make an appointment. At this time they can begin to call number inquiry (according to the order of registration). During the consultation, patients can not only communicate with each other through audio and video, but also view the patient's historical Internet hospital treatment data. After completion, the doctor can make consultation suggestions or diagnostic suggestions. Diagnostic suggestions include diagnosis, treatment, drug prescription, inspection and examination, etc., which can only be seen by the patient after the prescription is reviewed by the pharmacist, and the corresponding process can be completed.

\subsection{Internet Hospital Online Diagnosis and Treatment Platform}

Offered are Internet hospital consultation services on the Internet; Medical practitioners online part of the common diseases, chronic disease follow-up services. Promote the combination of online and offline, the interaction within and outside the hospital, the triage between superiors and subordinates, the connection between the entity and the platform, and guide patients to use online hospital (hospital official account, APP and other entrances) online follow-up service or consultation service after the easy one according to the patient's return visit needs in the hospital (and medical association).

\subsection{Establishment of Internet Hospital Prescription Circulation Platform}

Carry out the online prescription of some common diseases and chronic diseases after mastering patients' medical records; Open up the channel of prescription circulation in hospitals (and medical associations). Complete the establishment and review of electronic medical records of Internet hospital and the hospital, and conform to the relevant provisions of prescription management, so that registered physicians could issue online prescriptions for some patients with common 
diseases and chronic diseases under the condition of having the patient's medical records, and the prescriptions would be reviewed by registered pharmacists on an average basis. According to the needs of prescription circulation of patients in the hospital (and medical confederations) (non-medical insurance patients in the first stage, all patients in the second stage), the easy to use first and then the difficult to actively promote and guide patients to use Internet hospital prescription circulation services for the convenience of people.

\subsection{Construction of Internet Hospital Health Management Platform}

Provide online health consultation, chronic health management and telemedicine services. Guide patients in the hospital (and medical confederates) to use the Internet hospital platform for health consultation, chronic disease health management, family (private) doctor services, and second diagnosis and treatment services. Strengthen the promotion and publicity of Internet hospital, and expand the influence of Internet hospital in the country. Explore the new health management mode, and become a competitive incremental channel for the Internet hospital platform. The long-term development of the Internet hospital management platform will further promote the sustainable and healthy development of online medical services in the hospital and facilitate the optimization of medical resource allocation in hospitals.

\subsection{Setting up the Backstage Management of Internet Hospital}

In order to ensure the normal operation of Internet hospital, ensure the service quality of hospitals and doctors, and reduce medical risks, it is necessary to build an Internet hospital management background, whose main functions include: user rights management, financial inquiry management, operation data inquiry management, operation content management, drug distribution platform and other modules. The Internet hospital management background can connect with the supervision platform, express delivery and other business systems, and provide unified entrance and services for the hospital management departments.

\subsection{System Safety Construction}

In the network communication, in addition to using HTTPS protocol to encrypt the data, the Internet hospital also adopts the secondary data encryption processing for the sensitive information of users. It realizes the encryption of the whole data of communication between the mobile phone terminal and the server application, effectively protecting the security of medical data in the network [9, 10]. The system carries on the special treatment to the user's password and the related sensitive information, prohibits to save in the client. When sensitive information is displayed in the $\log$ and stored in relevant data tables, replacement processing is adopted to enhance the confidentiality and security of user information during business processing. The system keeps track of the whole process and records the transaction log information in detail, such as the transaction time, user, mobile platform, client version, user location, transaction category, transaction result, remarks information, etc., to provide data support for the transaction log verification.

\section{Thoughts on the Development of Internet Hospitals}

The development of Internet hospitals is in line with the national goal of deepening the reform of the medical and health system by the development of public hospitals, and can effectively promote the implementation of hierarchical diagnosis and treatment [11]. The cross-border integration of all kinds of clinical medical services and the Internet is an inevitable trend of the development of the medical industry in the current information age, and it will also be a hot spot at this stage $[12,13]$. Internet hospitals are convenient for patients, make full use of the high-quality medical resources of large public hospitals. They expand the social influence of doctors and hospitals, and actively adapt to the trend of the reform of hierarchical diagnosis and treatment in the national health system [14]. By giving full play to the enabling advantages of Internet hospitals, it will bring positive significance to patients, doctors, hospitals and health administration departments $[15,16]$.

The hospital has made some achievements in the practice of Internet hospital construction, but has also encountered the bottleneck problems faced by other Internet medical services in promoting the accessibility of Internet hospital services [17]. First of all, the income distribution structure is not compatible with the Internet medical services, and the online audit and settlement channels of the Internet medical insurance are not smooth; Secondly, the electronic health card, electronic social security card and medical insurance electronic voucher information are not interlinked; Finally, the list of common diseases and chronic diseases that can be deal with by Internet hospitals is not clear enough. Therefore, suggest that countries in line with the pricing mechanism of the development of the Internet hospital, set up a multiple card fusion mechanism from the top-level design, realize the card (yards) communication, open Internet health online audit and settlement channels, further refine the Internet regulatory standards, norms, hospital services to develop Internet medical services guidance document [18]. With the arrival of $5 \mathrm{G}$ era, Internet hospitals have entered a new stage of development. With the further promotion of national policies, Internet + pharmacy clinic and Internet + ultrasound clinic will also be included in the closed-loop process of Internet medical treatment to provide more convenient and efficient diagnosis and treatment services for people [19].

Internet hospitals break the time and space limitation of traditional medical services and provide patients with borderless closed-loop medical services. Although Internet hospitals can provide patients with medical services more conveniently, a number of diagnosis and treatment services 
still cannot be carried out online. For example: Diagnostic services for patients with first diagnosis, critical illness, and auxiliary diagnosis such as examination and examination, the prescribing and distribution of narcotic drugs and psychotropic substances cannot be carried out through the Internet. Whether the first diagnosis of common diseases should be allowed in the future, as well as the multi-site practice of doctors, remains to be explored and broken through by the policy [20]. With the continuous improvement of policies and the maturity of application technologies, the functions of Internet hospitals will become more practical and convenient. In the new era, more convenient, efficient and safe online diagnosis and treatment services will be provided to the public, so that people, including those in remote areas, can enjoy the dividends brought by the development of Internet hospitals.

The authors believe that the future development trend of Internet hospitals in the future will include the following three aspects: First, due to the rapid growth of China's aging population and the gradual increase in the incidence of chronic diseases, Internet + chronic disease management will become the focus of follow-up construction. Second, The Internet hospital can realize the advance of medical service from the family doctor service to providing patients with daily physical examinations, life and rest management, disease prevention and treatment guidance, etc. Health management will become a new model of the Internet hospital in the future. Third, With the development and maturity of $5 \mathrm{G}$, the Internet of Things, virtual reality technology, artificial intelligence, block chain and other technologies, the process of hospital digitalization will be significantly accelerated, technological change will push the application of health big data, and telemedicine will be widely applied.

\section{Conclusions}

The development of Internet + medical treatment is fundamentally a change in the mode of doctor-patient service. The construction and development of Internet hospitals can help revitalize the stock medical resources, improve the accessibility of quality medical services, and alleviate the difficulties in seeing a doctor. In practice, Hospital $J$ has explored a way to develop an Internet hospital in line with the characteristics of itself. The carrying capacity of Internet hospitals basically covers all links of patients before, during and after diagnosis, and plays a role in improving quality and efficiency in some procedures.

With the continuous development of our country's economy, the increasing demands for medical services, although the Internet hospital or new things, but it is used to supplement the hospitals, greatly improve the efficiency of medical service, easing the line, the queuing, medicine problem such as line, saves the time of both patients and medical staff. In future, with the continuous development of technology, Internet hospitals still offer much room for improvement and need continuous exploration. Providing high-quality and convenient diagnosis and treatment services for patients through Internet hospitals will certainly become an inevitable trend in the development of the medical industry in the future.

\section{References}

[1] Zhou Yuanyuan, Chen Dafang. Analysis and Suggestions on the Status Quo of Legal and Policy Protection in "Internet + Medical and Health Care" [J]. Chinese Journal of Cancer Prevention and Treatment, 2020, 12 (6): 606-610.

[2] Wang Peng, Wu Hao. Discussion on the current situation and future development trend of mobile Internet medical application at home and abroad [J]. China Digital Medicine, 2014, 9 (1): 8-10.

[3] Zhang Lei. Practice Research of "Internet + Medical Care" Led by Public Hospitals -- Taking Weifang Hospital of Traditional Chinese Medicine as an Example [J]. Journal of Traditional Chinese Medicine Management, 2019, 27 (21): 10-12.

[4] Wang Xiaobo, Li Fan. Current situation and regulation of Internet hospital development in China $[\mathrm{J}]$. Health Economics Research, 2020, 37 (11): 23-25.

[5] Zhang Mengqian, Wang Yanhuan, Qian Zhenguang, et al. Analysis on the development mode of Internet hospital in China [J]. Journal of Health Economics, 2019, 36 (5): 24-26.

[6] Zhou Li, Wu Qinqin, Liao Banghua, et al. Operation status and development ideas of Internet hospitals [J]. China Hospital Management, 2019, 39 (11): 59-62.

[7] Ding Ning, Hu Yu, Zhang Ming, et al. Current situation and development path of Internet hospital construction in China [J]. Chinese Journal of Hospital Management, 2020, 36 (1): $1-4$.

[8] Sun Guoqiang, You Lian, Chen Si. A preliminary exploration of Internet + medical model [J]. China Digital Medicine, 2015, 10 (6): $15-18$

[9] Hu Jianping, Gao Xiaofei, Liu Juan, et al. Hospital information security and supervision platform on mobile Internet $[\mathrm{J}]$. Chinese Journal of Health Information Management, 2015, 12 (1): 14-19.

[10] Xue Yifeng, Zhao Qi, Wang Yanli, et al. Design and implementation of mobile Internet hospital cloud platform [J]. China Digital Medicine, 2015, 10 (1): 94-95.

[11] Wei Mingyue, Cui Wenbin, Wang Shu, et al. Risk analysis and control strategy of Internet hospital [J]. China Health Resources, 2020, 23 (2): 99-101.

[12] Xiao Xing. Construction, Management and Effectivity of Palm Hospital System Based on "Internet $+"[\mathrm{~J}]$. Computer Programming Skills and Maintenance, 2020, 10 (1): 98-100.

[13] Ren Yufei, Tuo Bingbing, Yang Chong, et al. Construction practice of online and offline integrated Internet hospital cloud platform [J]. Chinese Journal of Hospital Management, 2020, 36 (10): 837-840.

[14] Guo Yan. Promoting the construction of Internet hospital and helping the whole people's health [J]. Chin J Critical Care Med, 2020, 32 (7): 771-773. 
[15] Zhang Lijiang, Zhao Xiaoxiao, Gui Liang. Discussion on the improvement path of doctor dispatching management in Internet hospital [J]. Journal of Hospital Management, 2020, 36 (10): 829-832.

[16] Zhu Haiyan, Zhang Linyi, Yang Xiaojun, et al. The exploration and preliminary practice of the hierarchical medical services in the internet-based hospital model [J]. Chinese Health Standard Management, 2021, 12 (5): 9-13.

[17] Luan Yunbo, Tian Zhendu. The existing problems and countermeasures of "Internet medical treatment" in China [J]. Administrative Management Reform, 2017, 9 (3): 59-63.
[18] Hu Huaining, Gu Hongli, Geng Yang, et al. Exploration and practice of chronic disease management mode in Internet hospital [J]. Journal of Nursing Management, 2021, 21 (2): 144-147.

[19] Yu Mengfei. A Brief Analysis of the Development Status and Prospect of Internet Hospital [J]. Network Security Technology and Application, 2021, 12 (1): 131-133.

[20] Yuan Weizhou, Wang Cuiping, Lin Xiwen, et al. Construction and application of Internet hospitals in the new era [J]. Journal of Medical Informatics, 2020, 41 (10): 57-59. 